\section{Substantia granulofilamentosa}

H. Baum

Institut für Laboratoriumsmedizin, Mikrobiologie und Blutdepot, Regionale Kliniken Holding RKH GmbH, Ludwigsburg, Deutschland

Englischer Begriff substantia granulofilamentosa

Definition Konglomerat aus Ribonukleoproteinen und einem Vitalfarbstoff in Retikulozyten.
Beschreibung Die Substantia granulofilamentosa ist ein morphologisch nachweisbares Produkt der Reaktion von Ribonukleoproteinen des jungen Erythrozyten ( $\triangleright$ Retikulozyt) und einem Supravitalfarbstoff, wie z. B. Brillantkresylblau oder Nilblausulfat. Ihr Nachweis dient der Erkennung und Zählung der Retikulozyten.

\section{Literatur}

Enne W (1993) Zellen der Erythropoese. In: Begemann H, Rastetter J (Hrsg) Klinische Hämatologie, 4. Aufl. Georg Thieme Verlag, Stuttgart, S 35-36 